\title{
Use of Social Media among Intern Doctors in Regards to Covid-19
}

\author{
*Prativa Subedi', Bibechan Thapa ${ }^{1}$, Aakriti Pandey ${ }^{1}$
}

Author Info:

${ }^{1}$ KIST Medical College and Teaching Hospital, Lalitpur, Nepal

\begin{abstract}
Background: The world is not only fighting a pandemic of COVID-19, but also tackling an infodemic due to rampant spread of misinformation and rumor about COVID-19 across various online media. Social media is easy, quick and costeffective source of information but unfortunately its contents are not regulated. Users usually do not verify information on social media on authentic sites. So, misinformation spreads exponentially. Intern doctors as young medical trainees should have scientific knowledge of disease and not be misled by rumors. Furthermore, health related information shared by medical professionals including interns owes greater credibility for public. This study aims to explore whether or not intern doctors are using social media rationally in regards to COVID-19.
\end{abstract}

Methods: This is a cross sectional, mixed method study carried out among medical and dental interns of KIST Medical College and Teaching Hospital. Data was collected from 27 Feb-24 March, 2020 with the help of self-administered semi-structured questionnaire and two Focus group discussions conducted on medical and dental interns respectively.

Results: $65 \%$ intern doctors most often used social media to gain information about COVID-19 of which $87.1 \%$ mostly used Facebook. Around $46 \%$ just read the full title of news appearing in social media and $36.5 \%$ shared the news just by seeing title. Most of the interns agreed that social media had spread rumor and information about COVID-19 but only $35.3 \%$ intern doctors check its authenticity by verifying it via experts or from authentic sources

Conclusion While intern doctors agreed to the notion that social media spreads rumors and misinformation, the verification rate of news related to COVID-19 on authentic sites is low among themselves. Furthermore, they also share unverified, non-evidence based information on social media, thus contributing to the infodemic. Hence, their use of social media doesn't seem rational.

Keywords: Authenticity, Coronavirus, COVID-19, Infodemic, Rumor, Social Media

Article Info
How to cite this article in Vancouver Style?
Subedi P, Thapa B, Pandey A. Use of Social Media among Intern Doctors in Regards to Covid-19.
Europasian J Med Sci. 2020;2(1):56-64
https://doi.org/10.46405/ejms.v2i1.41




\section{INTRODUCTION}

COVID-19 is an emerging infectious disease caused by novel coronavirus that started as an outbreak in Wuhan, China on December, 2019, declared as Public Health Emergency in 30 January 2020, and was declared pandemic on $11^{\text {th }}$ March, 2020by World Health Organization (WHO). Total number of confirmed cases were 87, 137 on $1^{\text {st }}$ of March, 2020 which rose to $12,10,956$ by $6^{\text {th }}$ April, $2020 .{ }^{1}$ With steep rise of epidemic curve, there has been surge of unprecedented level of information as well as misinformation regarding COVID-19 on social media. Social media refers to forms of web based media that allows interactive participation. ${ }^{2}$ The commonly used social media in Nepal are Facebook, Youtube, Instagram, Viber and Twitter. ${ }^{3}$ As evidenced by researches, roughly $45 \%$ of the world's population use social media and spend an average of 2 hours and 23 minutes per day and about $32 \%$ of office hour (productive time) on social media. ${ }^{4}$ Such significant engagement in social media clearly explains its influence on the thought process ${ }^{5}$ and perception of an individual leading to a change in behavior. It thereby contributes to the dynamics of rapid spread of misinformation.

As scientists are striving to find a cure for this disease, WHO and other health agencies are also working to identify potentially harmful rumors on COVID-19 and refute it with evidence-based information in order to combat this infodemic. ${ }^{6}$ Various myths have been circulating in regards to the clinical and epidemiological profile, diagnosis, prevention as well as treatment of this disease. ${ }^{7}$ With misinformation and rumors spreading rapidly across various platforms on social media, it has created a state of fear, frustration and stigma Likewise, adoption of unscientific measures to contain the epidemic is on rise. Although social media is widely used, its contents are not well regulated; and information on social media are usually not verified by users on authentic sites. So, misinformation and rumors continue to propagate rapidly, that not only renders millions of people vulnerable to wrong practices but they also fail to absorb recommendations that are scientific.

Social media use is found to be highest in the age group 18-29 years. ${ }^{8}$ As intern doctors also fall under the same age group, they are highly active in social media. Interns are young physicians in training who have a medical degree but can only work under supervision. ${ }^{9}$ They have to encounter COVID-19 patients and are also expected to answer COVID-19 queries of patients, patients party as well as other acquaintances of their own. So, it is essential for them to have scientific and authentic information regarding COVID-19 and not be misled by social media rumors. Likewise, information shared by them owes greater credibility to public. So, it is important that they use social media rationally and responsibly and promote a culture of authentic news sharing in the light of COVID-19 pandemic.

\section{MATERIALS AND METHODS}

This is a cross sectional, mixed method study conducted among intern doctors of KIST Medical College Teaching Hospital, Nepal. Quantitative data was first collected using self-administered semi structured questionnaires. In order to gain in depth formation, qualitative study was done with the help of Focus Group Discussion. All the interns currently pursuing their internship and willing to participate in the study were enrolled in our study. None of them declined participation. Reliability and validity of questionnaire was tested by pretesting on $10 \%$ the population (95) i.e. ten interns. Pretested individuals were not included in the final survey and were requested not to disclose any information regarding our study to other interns so as to minimize bias. Remaining 85 interns including 57 from MBBS and 28 from BDS participated in the final survey. Data collection was done from $27 \mathrm{Feb}$ to 9 March 2020. During this period, disease was in the phase of early epidemic. Descriptive statistics in terms of frequency and percentage were used to analyze the results of our study. The analysis was done using the SPSS 16.0

In order to gain detailed information regarding the data collected in survey and with a view to reduce the information bias, two FGDs were conducted using FGD guidelines separately among medical interns (FGD1) and dental interns (FGD2). Validity and reliability of FGD guidelines was tested via expert opinion. Ten participants from medical interns and eight participants from dental interns were purposefully selected for FGDs. Gender wise distribution was equal in both FGDs. Considering the state of lockdown and to abide by the norms of social distancing, FGD was conducted via videoconference. FGDs were conducted on 23th and 24th of March 2020, whereby COVID-19 had already been declared a pandemic. Ethical approval was taken from Institutional Review Committee of KIST Medical Teaching Hospital. Written consent 
was taken from all the participants for the survey whereas verbal consent was taken for FGD. Participants were not provided any incentive for being enrolled in the study. All data access was limited to authors involved.

\section{RESULTS}

Total of 85 intern doctors were enrolled in our study which comprised of $51.8 \%$ females and 48.2 $\%$ males. Medical interns constituted around two third $(67.1 \%)$ of the study population while dental interns constituted the remaining one third (32.9 $\%)$. Age of the participants ranged from 20 to 28 years with a mean age of 24.74. Similarly, $82.4 \%$ (70) intern doctors had first heard about COVID-19 from social media followed by friends/ family/ relatives (10.6\%), TV (2.4\%), Newspaper (2.4\%), and Experts (2.4\%). All the 85 participants mentioned that they had read or searched about COVID-19 on internet/ online media. Out of them, $63.5 \%$ (54) mentioned that they read/searched about it at least once a day. Amongst various sources on internet, $65.5 \%$ of the intern doctors most often used social media to gain information about COVID-19. Only $16.9 \%$ used official websites of health agencies like WHO for acquisition of information on COVID-19 (Table $1)$.

Amongst various social media platforms, $87.1 \%$ (74) interns used Facebook followed by Youtube (7.1\%), Instagram (4.7\%) and Twitter (1.2\%) to gain information about COVID-19 (Figure 1). Only $32.9 \%$ (28) intern doctors read the full text of the news appearing in social media while $45.9 \%$ (39) just read the title. Remaining 21. 2\% (18) mentioned that they would do both, actions varying on

Table 1: Most often used internet source to gain information about COVID -19

\begin{tabular}{|l|l|}
\hline $\begin{array}{l}\text { information about COVID -19 } \\
\text { Source }\end{array}$ & $\begin{array}{l}\text { Number of intern } \\
\text { doctors }\end{array}$ \\
\hline Social media & $56(65.88 \%)$ \\
\hline $\begin{array}{l}\text { Authentic health } \\
\text { sites like WHO, CDC }\end{array}$ & $14 \quad 16.47 \%)$ \\
\hline Online newspaper & $8 \quad(9.41 \%)$ \\
\hline Wikipedia & $5(5.88 \%)$ \\
\hline Others & $\begin{array}{l}2 \text { (2.35 \%) } 1 \text {-Medscape } \\
1-U p t o d a t e\end{array}$ \\
\hline
\end{tabular}

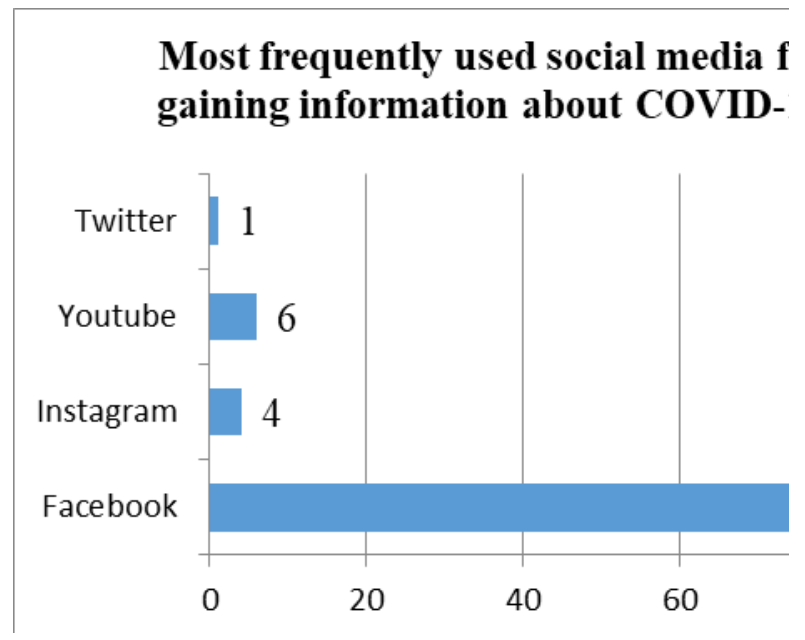

Figure 1: Most frequently used social media for gaining information on COVID-19

different occasions. The study participants were asked whether they have shared news on social media just by seeing title. To this, 36.5 (30) intern doctors mentioned that they had done so. Only $35.3 \%$ (30) intern doctors check the authenticity of information on social media by verifying it via experts or from authentic sites. Difference was found between medical and dental interns. That is $40.4 \%$ (23) medical interns and $25 \%$ (7) dental interns mentioned that they check the authenticity of information on social media (Figure 2). About $62.4 \%$ (53) intern doctors had shared news or written posts about COVID-19 in social media. Amongst them $81.2 \%$ (43) shared it at least once a day (Figure 3).

In terms of sharing, $41 \%$ of intern doctors and 66 $\%$ of those who share information on social media i.e. 35 interns share it randomly without verification (Figure 4). Verification rates before sharing were found similar among dental interns (34.3\%) and medical interns (33.3\%) (Figure4).

In context of using the social media information in practice, $50.6 \%$ (43) verified it before using it for their daily life and $67.1 \%$ (57) verified it before using it in terms of patients. Ninety-four percent intern doctors (80) felt that social media spreads more rumors than facts about COVID-19. But only $14.1 \%$ (12) had reported or discouraged such news

Around $89 \%$ (76) believed that news shared by them in social media creates more impact on public than a non-medical person and $92.9 \%$ (79) intern doctors believed that it is their responsibility to share only authentic information. Meanwhile, $34.1 \%$ (29) admitted that they had contributed 


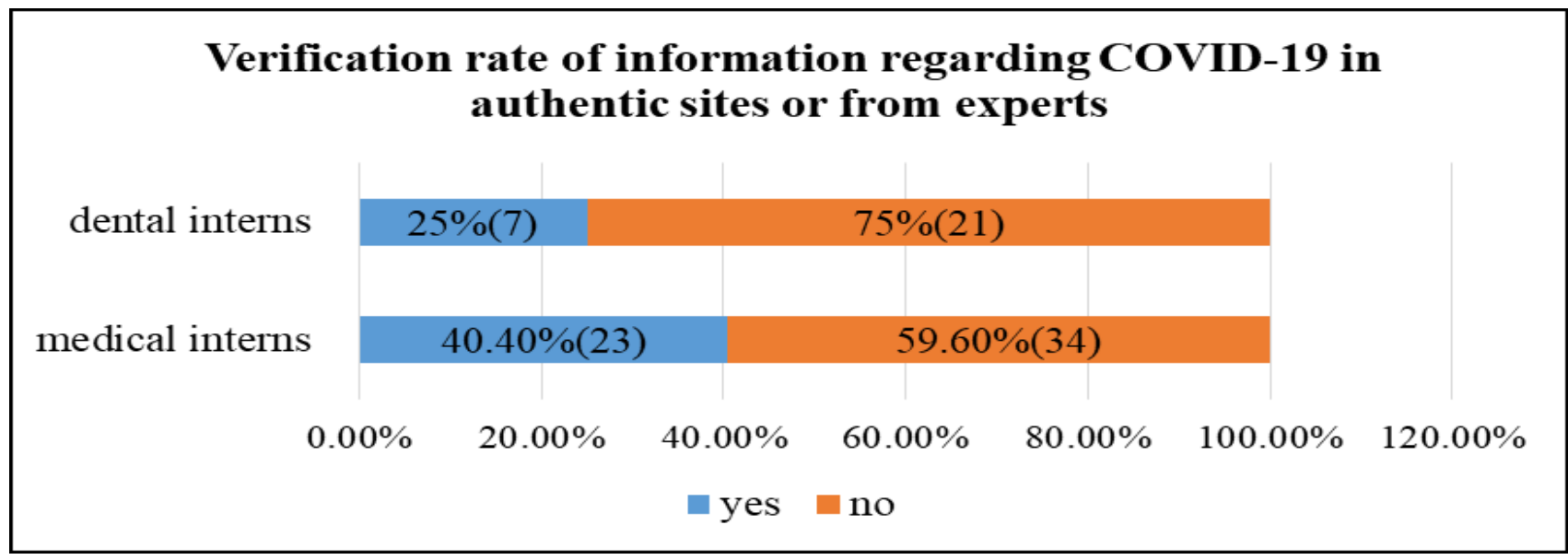

Figure 2: Verification rate of information regarding COVID-19 among medical and dental interns in social media.

\section{Frequency of sharing news/information about COVID-19 in social media}

\begin{tabular}{|c|c|c|c|}
\hline \multirow{3}{*}{$25(29.4 \%)$} & & & $32(37.6 \%)$ \\
\hline & \multicolumn{2}{|l|}{$18(21.2 \%)$} & \\
\hline & \multicolumn{2}{|c|}{$10(11.8 \%)$} & \\
\hline More than once a day & once a day & rarely & never \\
\hline
\end{tabular}

Figure 3: Frequency of sharing news/information about COVID-19 in social media

in spreading rumors of any kind about COVID-19 through social media. A $77.6 \%$ (66) intern doctors agreed to the opinion that social media has played major role in spreading terror in general public.

\section{FOCUS GROUP DISCUSSION FINDINGS:}

In case of an emerging disease, the disease pattern is unpredictable and keeps changing. With this, people's perception and behavior of people in response to the outbreak changes too. We therefore conducted our FGD two weeks after our survey so that we could compare two scenarios too.

FGD participants were asked how often they read/ searched about COVID-19. All of them mentioned that they searched/read about COVID-19 at least once a day and agreed that the frequency had increased compared to the survey time. When they are asked about the potential factors associated with this change, following responses were received-

"We were not much bothered and did not use to search much about COVID-19 earlier, but now that the cases are rising at an alarming rate all over the world, we constantly check the statistics." - FGD1 participants

"Lockdown has given us so much of free time. As we are staying at home all day and we have nothing much to do, we search and read about COVID19 quite a lot."-FGD2 participants

Participants were asked about the need of verification of social media information regarding COVID-19 on authentic sites. Following responses were obtained from the participants.

"We can hardly believe what is written in social media. COVID-19, being such a sensitive issue that has indeed become a pandemic and we, being 


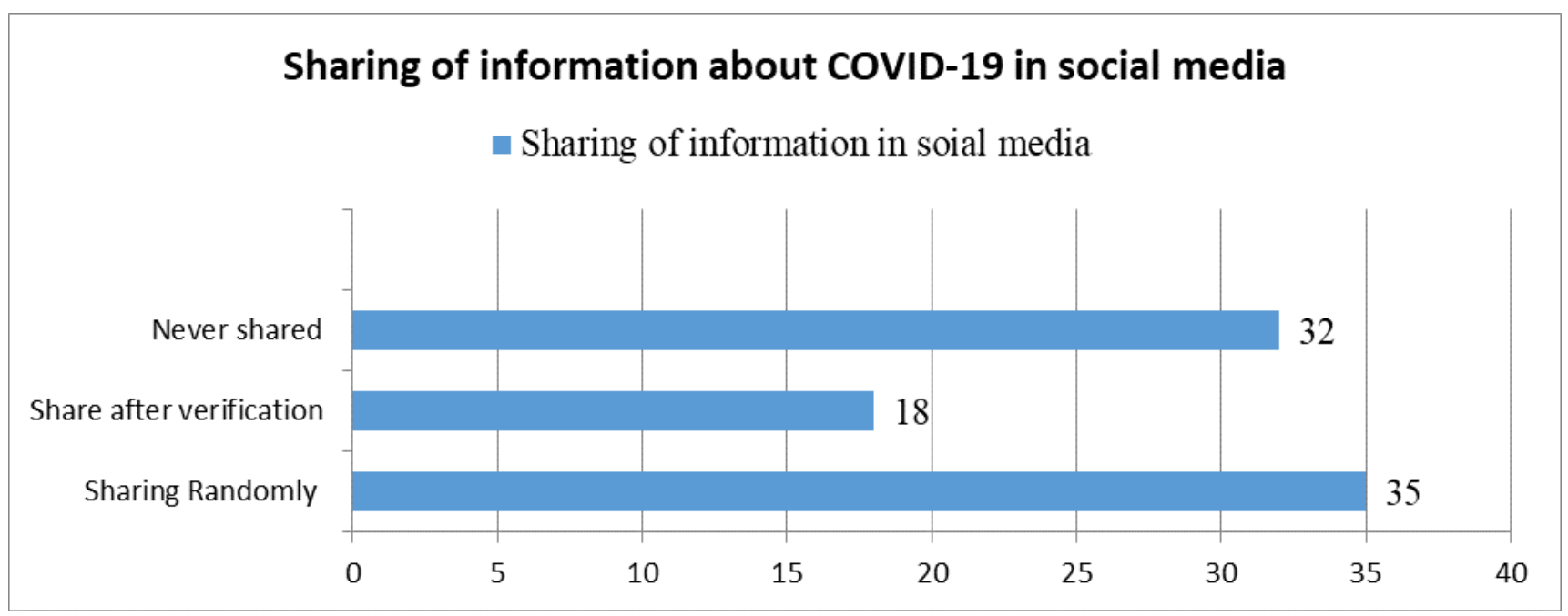

Figure 4: Sharing of information about COVID-19 in social media

interns, it is absolutely necessary to verify the information. It is better to search on Google. It can be checked on official sites of health agencies like WHO, CDC too."- FGD1 participants

"It is not necessary to verify each and everything. Social media says that eating garlic and turmeric prevents from COVID19. It may not be true. But there is no harm them, isn't it? But yes, if social media claims of a new cure like medicine or a vaccine, that has to be verified"-FGD2 participants

Participants were asked if they read the full news and watched the full videos about COVID-19 on social media and enquired about the factors that made them read the full news or watch the full video. Participants mentioned that they did either depending on the title and source.

"I think we Nepalese are driven by emotional content. The videos that are trending in Youtube are usually those which are emotionally charging both by their thumbnails and the content. It holds true in case of COVID-19 too. That news with sensational titles have the maximum views and shares."- FGD1 participants

"Almost all of the posts in social media right now are about COVID-19. It is impracticable to completely watch or read them all. Titles that are catchy and seem to deliver new information provoke the readers or audience to go through whole of it or at least half of it"-FGD2 participants

FGD participants were asked about the authenticity of news they had shared in the social media.

"While sharing or writing a public post regarding
COVID19 in social media, we tend to verify it in authentic sites. But in closed groups, personal chat or group chat, we usually share it randomly. It is not possible to verify each and every news."-FGD1 participants

"Authenticity is not clearly defined in the context of COVID-19. Since it is an emerging disease, nobody is an expert on it. So, sharing news is rather a trust issue. Apart from $\mathrm{CDC}$ and $\mathrm{WHO}$, information on national dailies are quite trustworthy. News posted by a Routine of Nepal Banda [a popular social media page]. are also mostly correct posts. We tend to share posts by pages and people that we trust"-FGD2 participants

Participants' views were asked about the spread of rumors about COVID-19 by social media and asked if they themselves had contributed in spreading of any kind.

"It should be rather called misinformation than rumor. Social media spreads information that are partially right and partially wrong. We might have not contributed directly. But indirectly or unwillingly, we might have contributed in the spread. "- FGD1 participants

"In view of getting easy popularity, many people or pages are spreading news with COVID-19 cases being positive in random people when it is actually not. These rumors are the major keys for the fear and anxiety among people"- FGD 2 participants

FGD participants were asked if they had discouraged or reported such news spreading rumors and asked why they had not. Some participants mentioned that they felt that the rumors were not very harmful 
enough to prompt reporting and mentioned that they had personally discouraged their friends if they spread rumors that were potentially harmful like 'alcohol consumption' could cure COVID-19. Meanwhile, some participants mentioned that they did not report or discourage such posts because they did not want to get into any conflict.

Participants were asked about their view regarding the spread of terrors. To this, the participants responded that

"Yes, social media spread a lot of terror. Every now and then, people are sharing the number of deaths and cases. News that COVID-19 is more in old and comorbid people has caused most of our grandparents to be really anxious "- FGD1 participants

"During early outbreak, we felt that social media is spreading a lot of terror. But now that we have a case in our country too, a level of terror seems to be necessary. It has caused people to follow quarantine and self-distancing rather strictly. It should be taken positively now."- FGD2 participants.

When FGD participants were asked about how they used social media information, they answered that they were more cautious and checked the authenticity of information while using any information in practice.

"For COVID-19 patient triage and management, we generally follow hospital protocol rather than social media. Most of the preventive measures that we use in practice are similar to that of other flus. But for use of PPE, we go through authentic sources, rather than just relying on social media posters. So many doctors are dying in China, we cannot take it lightly "- FGD1 participants

"Yes, we try to be more authentic while using it in practice. We are more cautious when it is related to patients. We have never ever counseled any patients regarding the use of chloroquine, despite many social media proclaiming its effectiveness. It is better not to speak, if we are not completely sure of it. "-FGD2 participants

\section{DISCUSSION}

With 3.8 billion social media users' worldwide, social media constitute the largest platform for idea and information sharing. ${ }^{10}$ Facebook was found to be the most frequently used source of information regarding COVID-19 among interns in our study population. Facebook indeed is the largest social media platform with 2.5 billion users worldwide as of Jan, $2020 .{ }^{10}$ In context of Nepal, a digital research done in April, 2019 showed that there were 9,827,000 active Facebook users, which accounted for $32.4 \%$ of its entire population. ${ }^{11}$

With the rise in number of cases and rapid spread of COVID-19 in countries other than China, it has been the major concern of people all around the globe. As of March 12, coronavirus has seen its highest level of search traffic — that level is indicated by a score of 100 on Google Trends and 19 million mentions across social media and news site in 24 hours. $^{12}$ During the early stages of disease outbreak, there generally is great deal of uncertainty about the facts. Confirmation of facts is not always immediately possible in case of emerging diseases and scientific information and peer reviewed-literature is always lagging behind the news report. ${ }^{13}$ Thus people are largely reliant on social media for information. Since information about emerging infectious diseases on social media are user generated, they are most often based on self-assumptions and speculations rather than on scientific grounds. ${ }^{14}$ People can deliberately spread misinformation and racism. With an incredible speed and reach, information in social media can spread like a contagious epidemic. The process of transmission and retransmission across social media allows misinformation and rumor to spread at an exponential rate. This can be related to an epidemiological model of Reproduction number (Ro) as explained by M. Cinelli et al. ${ }^{15}$ James Holland Jones from Stanford university has defined Ro as" The expected number of secondary cases produced by a single (typical) infection in a completely susceptible population." . ${ }^{16}$ A disease with Ro $<1$ dies out over finite period of time, while the one with $\mathrm{RO}>1$ is highly contagious and can cause an epidemic. Likewise Ro> 1 in social media can cause infodemic. ${ }^{15}$ So, it is very important that the information which circulates in the social media regarding COVID-19 be an accurate and has scientific basis

Health professional are at great risk of contracting COVID-19 during their treatment process. According to an article in New York Times, the exposure is highest amongst dentists. ${ }^{17}$ This could be due to their closed and long hours of work around the oral cavity. Other physicians also have 
to work in close proximity with COVID-19 patients. So, it is important for dental and medical interns to have adequate and authentic information about COVID-19. But Only $16.9 \%$ intern doctors were found to use authentic sites like official sites of $\mathrm{WHO}, \mathrm{CDC}$ as the primary source of information while majority of them (65.9\%) relied on social media information about COVID-19. If this is the figure in case of young physicians, it is likely to be less authentic and more social media based in case of general people. Easy and quick access to social media and inadequate information on authentic sites of health agencies and paucity of scientific researches and literature seem to be the major reasons for their reliance on social media information during early outbreak. Intern doctors during FGD session opined that it is hard to find adequate information about COVID-19 on authentic health sites too which is the reason for them to be social media dependent. The situation, in this regard is improving with regular update of information and initiation of various online courses started by health agencies like WHO, medical universities, national and international organization in order to boost the information on COVID-19. Free and rapid access to high-quality information from verifiable sources is valuable to optimize the global medical response to crises like COVID-19 pandemic. $^{18}$

In case of emerging disease, where enough is not known about the disease, public's knowledge about the disease is largely based on the information being circulated across various print and social media. News are often fabricated in social media and manipulated for easy popularity. Clickbaits withhold crucial information and contain intriguing, emotional and fallacious content. ${ }^{19}$ In our study,36. 5\% intern doctors share news and videos just by reading the title and $41.2 \%$ share it randomly without verification. FGD participants also mentioned that they are more liberal and less cautious while sharing information about COVID-19 in social media in closed groups and personal chats. This allows misinformation and rumor to propagate across the invisible, fast and furious limbs of social media.

Most of the interns believe that social media has spread rumors about COVID-19. But verification rate of social media information by interns is found to be low in our study, be it in the context of sharing the information or using it in daily practice. Moreover, the tendency of reporting or discouraging such information is also considerably low (14.1\%). Just like a poison can do much harm, if antidote is not administered. Misinformation in social media can do a lot of harm, if they are not refuted or counteracted.
There are many instances of it where rumors spread in social media have been potentially harmful. Despite the fact that many clinical trials are underway in search of the vaccines and medications for COVID-treatment, in the absence of concrete evidence of its efficacy and safety, it is likely to do more harm than good. ${ }^{20} \mathrm{~A}$ man died in United States and her wife was in critical condition after they took the anti-malarial chloroquine phosphate they used clean their fish tank after Donald Trump tweeted it as a" Gift of God remedy for COVID-19". ${ }^{21}$ When influential people share such crucial information without checking its authenticity like this, public is gullible to such misinformation, thereby creating a mishap. On the contrary, social media can be a powerful source to spread useful information too. After WHO Director General urged all the people to join the \#SafeHandsChallenge and nominated few public figures, a lot of celebrities joined the campaign and posted videos of their handwashing on social media which is not only viewed but shared, reposted and retweeted by many people across the globe. ${ }^{22}$

Considering massive involvement of people in social media and the difference it can make, WHO emphasized the proactive use of social media to counteract misinformation on Emerging infectious diseases during E-bola outbreak. ${ }^{23}$ This holds significance in the context of COVID-19 too.

WHO has also collaborated with Facebook and What's app and launched Health Alert service so as to debunk the myths related to COVID -19 and help people get their queries answered. ${ }^{24}$ In reference to our study ,where Facebook is the most often used source of information about COVID-19 with $94.1 \%$ believing that social media spreads more rumors that facts $34.1 \%$ agreeing to have contributed to the spread of rumors, this can be considered an important step in combating the infodemic.

Most of the intern doctors have agreed that over flooding of information, especially the statistics of increasing deaths has spread a lot of terror among public. On the flip side of the coin, few interns also believed that the terror thereby created had made people more cautious and motivated them to take strict precautionary measures and practice social distancing.

Health related information shared by health professionals can have greater impact on the public than non-medical person. So, they must help to curb the spread of misinformation and make sure that reliable and authentic information reaches the public. Just as they practice and promote personal hygiene, they need to practice and 
promote 'information hygiene' and get COVID-19 information only from credible sources. ${ }^{25}$

\section{Limitations and Strengths}

Since this is one centered study carried out among intern doctors of single hospital, our sample size is small and the findings may not be very representative of all the intern doctors. Also the questionnaires are self-generated, so it might not have incorporated all aspects regarding rational use of social media. Mixed method with inclusion of FGD on the qualitative section however gives an edge to the study with reduced information bias.

\section{CONCLUSION}

Apparently, many of the intern doctors are obtaining information regarding COVID-19 from unauthentic sites on social media and they themselves have labelled most of the circulating news as 'rumors or 'misinformation'. But despite this notion, information verification rates are low and so is the rate of reporting misinformation, thereby exhibiting the irrational use of social media by intern doctors. Rational use of social media is one of the crucial measures to curb malpractices and anxiety related to COVID-19. Every individual, notably health workers, should be committed to authentic and evidence-based news searching, reading and sharing in social media to upgrade the knowledge related to COVID-19, maximize the precaution and minimize unnecessary panic. At the same time, social media content should be regulated. These measures can be crucial in combating the current pandemic of COVID-19.

Acknowledgements: We are thankful to all the participants involved in the study for their cooperation. We express our gratitude to Prof. Dr. Amita Pradhan, Prof. Madhusudan Subedi and Dr. Lila Bahadur Basnet for their guidance. We would like to thank all our anonymous reviewers.

\section{REFERENCES}

1. COVID-19 situation reports [Internet]. Who.int. 2018 [cited 2020 April 20]. Full Text

2. Manning J. Social media, definition and classes of. In K. Harvey (Ed.), Encyclopedia of social media and politics. 2014; 1158-1162). Google Scholar| Full Text

3. Prasain Krishana. Social media marketing shines in Nepal market [Internet]. @kathmandupost. The Kathmandu Post; 2018 [cited 2020 April 8]. Full Text

4. Ennis O' Connor M. How Much Time Do People Spend on Social Media in 2019? [Infographic]
[Internet]. Medium. 2020 [cited 2 May 2020]. Full $\underline{\text { Text }}$

5. Amgain K, Neupane S, Panthi L, Thapaliya P. Myths versus Truths regarding the Novel Coronavirus Disease (COVID-2019) Outbreak. J Karnali Academy of Health Sciences 2020;3(1):1-6. https://doi.org/10.3126/jkahs.v3i1.28367 Google Scholar| Full Text

6. United Nations [Internet]. United Nations; UN tackles 'infodemic' of misinformation and cybercrime in COVID-19 crisis. 2020 [cited 2020 April 20]. Full Text

7. Sajed AN, Amgain K. Corona Virus Disease (COVID-19) Outbreak and the Strategy for Prevention. Europasian Journal of Medical Sciences. 2020; 2(1):1-4.https://doi.org/10.46405/ejms.v2i1.38 Google Scholarl Full Text

8. Statista [Internet]. Statista. Statista; 2017 U.S. social network reach by age group 2017. [cited 2020 April 10]. Full Text

9. Wikipedia Contributors. Internship [Internet]. Wikipedia. Wikimedia Foundation; 2020 [cited 2020 April 16]. Full Text

10. Digital 2020: 3.8 billion people use social media - We Are Social [Internet]. We Are Social. 2020 [cited 2020 April 16]. Full Text

11. NapoleonCat. Facebook users in Nepal - January 2019 [Internet]. Napoleoncat.com. 2019 [cited 2020 April 16] Full Text

12. Rani Molla. Coronavirus dominates Facebook, Twitter, and Google searches [Internet]. Vox. Vox; 2020 [cited 2020 April 25]. Full Text

13. Allgaier J, Svalastog AL. The communication aspects of the Ebola virus disease outbreak in Western Africado we need to counter one, two, or many epidemics? Croatian Medical Journal. 2015;56(5):496.https:// doi.org/10.3325/cmj.2015.56.496 Google Scholar | CrossRef | PubMed | PMC | Full Text

14. Tang L, Bie B, Park SE, Zhi D. Social media and outbreaks of emerging infectious diseases: A systematic review of literature. American journal of infection control. 2018 Sep 1;46(9):962-72. https:// doi.org/10.1016/j.ajic.2018.02.010 Google Scholar| PMC) Full Text

15. Cinelli M, Quattrociocchi W, Galeazzi A, Valensise CM, Brugnoli E, Schmidt AL, et al. The COVID-19 Social Media Infodemic [Internet]. arXiv.org. 2020 [cited 2020 April 16]. Google Scholar| Full Text

16. Holland J. Notes on RO Department of Anthropological Sciences. Stanford University, Departament of Anthropological Sciences, Building. 2007;360:94305-2117. Google Scholar| Full Text

17. The Workers Who Face the Greatest Coronavirus Risk. The New York Times [Internet]. 2020 Mar 15 [cited 2020 April 16] Full Text 
18. Chan AK, Nickson CP, Rudolph JW, Joynt AL. Social media for rapid knowledge dissemination: early experience from the COVID-19 pandemic. Google Scholar| Full Text

19. Souppouris A. Clickbait, fake news and the power of feeling [Internet]. Engadget. Engadget; 2016 [cited 2020 April 16]. Full Text

20. Marahatta SB, Paudel S, Aryal N. COVID-19 Pandemic: What can Nepal do to Curb the Potential Public Health Disaster. Journal of Karnali Academy of Health Sciences. 2020 Feb 19;3(1):1-4. https://doi. org/10.3126/jkahs.v3i1.28374 Google Scholar| Full Text

21. Phoenix in. Arizona man dies after attempting to take Trump coronavirus "cure" [Internet]. the Guardian. The Guardian; 2020 [cited 2020 April 20] Full Text
22. Handwashing challenge [Internet]. Who.int. 2018 [cited 2020 April 20]. Full Text

23. SEA-CD-238 Distribution: General Developing tools for strategic communication to the media on emerging infectious diseases (EIDs) Regional Office for South-East Asia [Internet]. [cited 2020 April 15] Google Scholar| Full Text

24. Lacina L. WHO launches health alert service via Facebook and WhatsApp [Internet]. World Economic Forum. 2020 [cited 2020 April 25] Full Text

25. Zarocostas J. How to fight an infodemic. The Lancet. 2020 Feb 29;395(10225):676. https://doi. org/10.1016/S0140-6736(20)30461-X [Google Scholar| Crossref| PubMed| PMC| Full Text 